\title{
Feature Selection Using Genetic Algorithms for the Generation of a Recognition and Classification of Children Activities Model Using Environmental Sound
}

\author{
Antonio García-Dominguez ${ }^{\mathbb{D}},{ }^{1}$ Carlos E. Galván-Tejada ${ }^{\mathbb{D}},{ }^{1}$ Laura A. Zanella-Calzada ${ }^{(\mathbb{D}},{ }^{1}$ \\ Hamurabi Gamboa-Rosales $\mathbb{D}^{1},{ }^{1}$ Jorge I. Galván-Tejada, ${ }^{2}$ José M. Celaya-Padilla, ${ }^{1}$ \\ Huizilopoztli Luna-García, ${ }^{1}$ and Rafael Magallanes-Quintanar ${ }^{1}$ \\ ${ }^{1}$ Unidad Académica de Ingeniería Eléctrica, Universidad Autónoma de Zacatecas, Jardín Juárez 147, Centro, Zacatecas 98000 , \\ Zac, Mexico \\ ${ }^{2}$ CONACYT, Universidad Autónoma de Zacatecas, Jardín Juárez 147, Centro, Zacatecas 98000, Zac, Mexico
}

\begin{abstract}
Correspondence should be addressed to Antonio García-Dominguez; antonio.garcia@uaz.edu.mx and Carlos E. Galván-Tejada; ericgalvan@uaz.edu.mx
\end{abstract}

Received 30 August 2019; Revised 20 November 2019; Accepted 8 January 2020; Published 13 February 2020

Academic Editor: Raul Montoliu

Copyright (c) 2020 Antonio García-Dominguez et al. This is an open access article distributed under the Creative Commons Attribution License, which permits unrestricted use, distribution, and reproduction in any medium, provided the original work is properly cited.

\begin{abstract}
In the area of recognition and classification of children activities, numerous works have been proposed that make use of different data sources. In most of them, sensors embedded in children's garments are used. In this work, the use of environmental sound data is proposed to generate a recognition and classification of children activities model through automatic learning techniques, optimized for application on mobile devices. Initially, the use of a genetic algorithm for a feature selection is presented, reducing the original size of the dataset used, an important aspect when working with the limited resources of a mobile device. For the evaluation of this process, five different classification methods are applied, k-nearest neighbor (k-NN), nearest centroid (NC), artificial neural networks (ANNs), random forest (RF), and recursive partitioning trees (Rpart). Finally, a comparison of the models obtained, based on the accuracy, is performed, in order to identify the classification method that presents the best performance in the development of a model that allows the identification of children activity based on audio signals. According to the results, the best performance is presented by the five-feature model developed through RF, obtaining an accuracy of 0.92 , which allows to conclude that it is possible to automatically classify children activity based on a reduced set of features with significant accuracy.
\end{abstract}

\section{Introduction}

Artificial intelligence is an area in which there have been significant advances in recent years, caused largely by the evolution of technology. In this area, some subareas have had a greater development, as is the case of environmental intelligence [1], through which human beings seek to develop solutions that facilitate the activities of daily life and the interaction with their environment.

In recent years, with the growing development of environmental intelligence, different solutions and applications have emerged focused on providing services in different scenarios such as smart homes [2], smart buildings [3], smart cities [4], and other scenarios where it is possible to automate some type of interaction between people and their environment. Many of the works developed in the area of environmental intelligence are based on the concept of recognition and classification of human activities, through which it is possible to detect the type of activity performed by a person or group of people and offer a specific service or solution. This area of recognition and classification of human activities has had a huge boom in recent years, and there have been many works developed [5-10]. 
Within the works on recognition and classification of human activities, an important aspect is the group of people towards whom they are focused since different aspects such as the way to obtain the data and the services offered, among others, depend on it. Some works are directed to elderly $[11,12]$, others to children $[13,14]$, and most to adults interacting with defined environments [10, 15].

Another important aspect to be considered is the data source to be used, and numerous works have been developed using sensors as a data source $[16,17]$. In the area of recognition and classification of children activities, common data sources are video cameras, accelerometers, and radiofrequency devices $[18,19]$. Most of the works presented in this area perform data capture by placing the sensors directly on the children's clothing, which has the disadvantage that these sensors can interfere with children's natural behavior and alter the activities that they usually perform and that is intended to analyze. Also, as mentioned in the work of $\mathrm{Wu}$ et al. [20], this approach can be conducted based on one of the main roles and opportunities in the information and communication technologies nowadays, sustainable development goals, where the sustainable development in the acquisition of data can be performed with the purpose of assisting all nations in the development of research for multiple purposes.

The use of a data source that does not interfere with the performance of the activities to be analyzed is an alternative for the use of embedded sensors. Sound has been used as a data source $[21,22]$ with the advantage that its capture does not interfere with the analyzed activities because it is not necessary that the capture device is embedded in the clothing, and it can be at some distance from the place where the activity is being done. In addition, it is not necessary to use sensors or special hardware since it is possible to directly use the microphone of mobile devices for capturing ambient sound. For a general overview of a big data system development, the work of Atat et al. [23], where the different stages, including data collection, storage, access, processing, and analysis, are presented, develops a panoramic survey of working with large amount of data that are generated through a network.

To perform the process of recognition and classification of children activities using sound, it is necessary to select the audio features to generate a model that, trained with a part of the dataset, is able to predict to which class a given audio belongs. For the generation of these models, the genetic algorithm, Galgo (R package) [24], is implemented, based on a set of algorithms used as classification methods. The present work implements the k-nearest neighbor (k-NN), nearest centroid (NC), artificial neural networks (ANNs), random forest $(\mathrm{RF})$, and recursive partitioning trees (Rpart) classification methods, which are algorithms commonly used in recognition and classification of human activities [25-29].

Galgo implementation was selected given the availability to select relevant features that model the behavior of a phenomenon, even when it was developed in big search and bioinformatics applications. This algorithm has proven useful in various types of signals, such as magnetic field [30], complex systems [31], and features extracted from medical images [32, 33], to mention some.

In the work presented by Blanco-Murillo et al. [34], the generation of models for classifying children activities through environmental sound with the aforementioned algorithms is shown. These models were generated with a 34feature set extracted from the audios, and the classifier that achieves a higher precision is $\mathrm{k}-\mathrm{NN}$ with $100 \%$.

Previously, we presented a work where, with a larger dataset and the same set of extracted features, we perform a process of feature selection using the Akaike criterion, obtaining a 27-feature subset for the generation of the models with the same classification algorithms before mentioned, achieving accuracies greater than 90\%, with a $20 \%$ reduction in the size of the dataset due to the feature selection process, with the extra trees classifier obtaining the highest precision of $0.96 \%$.

In the present work, the same audio recordings and the same set of extracted features are used, but now performing a more efficient feature selection process, using the genetic algorithm, Galgo, [35], to generate recognition and classification of children activities models with the same classification algorithms and a reduced dataset, contained only by the most significant features of the activities analyzed, looking for the reduction in the size of the original dataset.

Therefore, the main question addressed in this work is the following: is it possible to develop a reduced features model for the classification of children's activities based on the environmental sound through the implementation of a genetic algorithm?

Being a work proposed for use and implementation by mobile devices, this reduction in dataset size positively impacts the processing time and resources of the devices since it works with a smaller amount of data.

\section{Materials and Methods}

The methodology of this work consists of four main stages, beginning with a brief data preprocessing, where the audio signals are divided in shorter time intervals. Then, a set of features are extracted from the audio signals, which are subsequently subjected to a feature selection based on a genetic algorithm. For the validation of this feature selection, five different classification methods are applied.

The feature extraction process is performed using the Python programming language [36], while the feature selection process and the model validation are performed using the R development environment and the Galgo library [24].

2.1. Data Description. The recordings used in the present work are taken from the previous work [37]. These recordings belong to four different activities, shown in Table 1.

2.2. Data Preprocessing. The data preprocessing consists on dividing the audio signals in ten-second time intervals. 
TABLE 1: General description of activities.

\begin{tabular}{lc}
\hline Activity & Description \\
\hline Crying & Emitting crying sound in reaction to some events \\
Playing & Handling plastic pieces \\
Running & Moving quickly from one place to another \\
Walking & Moving from one place to another at medium speed \\
\hline
\end{tabular}

2.3. Feature Extraction. For the feature extraction, a set of numerical features are extracted from the ten-second intervals of the audio signals, as shown in Table 2 .

These 34 extracted features are those that are commonly used in audio analysis and activity recognition activities through audio [38-41], especially the mel-frequency spectral coefficients, since many audio analysis works have been developed that make use of them [21, 42-44].

2.4. Feature Selection. The objective of the feature selection is to reduce the number of features by identifying those that present the most significant behavior in the classification of the audios. For this stage, the genetic algorithm (GA), "Galgo" [24], is implemented, using five different classification methods, in order to obtain subsets of five features. These classification methods consist of k-NN, NC, RF, ANN, and Rpart.

Galgo is a package developed under the R [45] language, implemented with an object-oriented approach and based on a general fitness function to guide the feature selection. The procedure of Galgo is designed to start with a random population of feature subsets, known as chromosomes. Each chromosome is evaluated measuring its ability to predict an output or a dependent feature, based on the accuracy. For this evaluation, a classification method is included. The main idea is to replace the initial population with a new one which includes features from different chromosomes that present a higher classification accuracy. This process is repeated enough times to achieve a desired level of accuracy. The progressive improvement of the chromosome population is performed by a process inspired on natural selection, based on three principles, selection, mutation, and crossover.

In resume, the application of Galgo consists of four main stages:

(1) Setting-Up the Analysis. In this stage, the parameters of the algorithm are set, including the input data, the outcome, the statistical model, the desired accuracy, the error estimation scheme, and the classification method, among others.

(2) Searching for Relevant Multivariate Models. This stage consists on the process of selection beginning with the random population of chromosomes, based on a classification method, looking for the best local solutions.

(3) Refinement and Analysis of the Local Solutions. Then, the chromosomes selected are subjected to a backward selection strategy, since, even when these chromosomes present the best accuracy, there could be features included in the model that does not
TABle 2: Extracted features.

\begin{tabular}{lc}
\hline Feature ID & Feature name \\
\hline 1 & Zero crossing rate \\
2 & Energy \\
3 & Entropy of energy \\
4 & Spectral centriod \\
5 & Spectral spread \\
6 & Spectral entropy \\
7 & Spectral flux \\
8 & Spectral rolloff \\
$9-21$ & MFCCs \\
$22-33$ & Chroma vector \\
34 & Chroma deviation \\
\hline
\end{tabular}

contribute significantly to the fitness value. The objective of this strategy is to derive a chromosome population containing only features that effectively contribute to the classification accuracy.

(4) Development of a Final Statistical Model. Finally, a single representative model is obtained based on a forward selection strategy, where according to a stepwise inclusion, the most frequent genes presented in the chromosome population are selected.

\subsubsection{Classification Methods}

(1) $k$-NN. This method has been widely used in different statistical applications. It presents a nonparametric approach, where its basis consists of, from a set of training data, a group of $k$ samples identified by being the nearest to unknown samples. To develop this process, the Euclidean distance, defined as $\|\vec{x}-\vec{y}\|$, between a given set of queries and the inputs, is calculated, identifying the $k$ closest input points for each query. Then, the output of the unknown samples is determined by calculating the average of the input features, based on the initial $k$ samples [46].

(2) NC. This method presents a partitional clustering approach, where data objects are grouped into $k$ clusters depending on their similarity. The parameter $k$ must be specified initially. The first selection of centroids is done randomly. To measure the closeness between a centroid and a data object, the Euclidean distance, cosine similarity, is calculated. Then, after this first grouping, a new centroid for each cluster is obtained, as well as the distance between each data object to each center, reassigning the data points according to the distance. The point in a cluster is treated as the centroid if the sum of all the distances between this point and all the objects of the cluster achieve the optimal minimization. The main objective of NC is to minimize the sum of distances between the objects of a cluster and its centroid [47].

(3) RF. This classifier was developed by Breiman et al. [48]. Its performance consists on two levels of randomness for the construction of the trees, beginning with a bootstrapped version of a set of training data, called bagging, where a 
subset of the training data is intended for each tree, based on the principle of replacement, while the remaining data are used to estimate the error, calculating the out-of-bag (OOB) error. Then, in the second level of trees, a subset of features is randomly selected and added to each node throughout the growth of the decision trees. In each node, the best feature is selected, looking for reducing the label error. This method bases its classification technique by the principle of the majority vote from all the decision trees. This process is recursively repeated until reaching a defined depth in the forest or the number of samples in a node does not exceed a threshold $[49,50]$.

(4) ANN. In this method, the searching for a specific task based on the correlation between features is performed. Its process consists on a learning or training that resembles the behavior of the biological neural networks. Through a series of different labels contain nodes or neurons, ANNs try to find a relational model between the input features and outcome. Three main elements are present in this method: (1) a set of synapses or connections characterized by a "weight," where the input signal is connected to a neuron through its product with the weight in that connection; (2) an adder, which aggregates the contributions of a signal pounded by all the weights; and (3) an activation function, equivalent to a transfer function, which is affecting the neurons, allowing to limit the amplitude of the outcome, providing a permissible range for the outcome signal in terms of finite values [51].

(5) Rpart. This is a statistical method for multivariate analysis which separate samples into different homogeneous risk groups to determine predictors of survival. The algorithm consists on selecting the predictor that provides the optimal split, such that each of the subgroups is more homogeneous with respect to the outcome. These subgroups are dichotomized into smaller and more homogeneous subgroups according to the feature that presents the best splits for each subgroup. A continuous iterative process is performed until presenting few values for additional splits. The pruning process is applied to the original partitioning tree in order to cut the tree back to the point where the overall predictive accuracy is maximized, preventing overfitting in the data [52].

\section{Results}

In Table 3, the number of recordings for each activity analyzed in the present work, as well as the number of tensecond clips generated after performing the corresponding division of the audios, is shown.

To perform the analysis, 34 features are extracted from each of the ten-second clips, consisting the dataset of 2716 samples.

Table 4 shows the general parameters of the genetic algorithm used in GALGO.

It is important to mention that, to select the chromosome size, the configuration presented by default by Galgo, which is five, was respected due to different points. First, because the size of the dataset is not very large, it was required to reduce the model to a size that would not compromise the performance of the classification and to avoid randomness in the development of the final model, with in order to test the robustness of the selection of features. On the contrary, this is the number that Galgo assigns by default if the user does not specify the size of the chromosome because, according to its description reported in the literature, this is the number of chromosomes that has presented the best performance for feature selection towards the different approaches. This is the same case for the selection of the number of niches [53].

Figures 1-5 show the performance of the fitness for each of the 200 generations evolved for the classification methods, k-NN, NC, ANN, RF, and Rpart, respectively. From these graphs, it can be observed from Figure 4, which correspond to RF, fitness (represented in the $Y$ axis) is the one with a higher accuracy from the beginning of the process, being stable in terms of the evolving process from the GA.

Figures 6-15 show the frequency of appearance of the features in the 300 models developed for the k-NN, NC, ANN, RF, and Rpart classification methods, respectively. These features presented in color black are the five who presented the highest frequency of appearance and, therefore, selected as the most representative. From these, low computational cost algorithms (as k-NN, Rpart, and NC) acquire a stable frequency (shown in the $Y$ axis) for the selected features, while complex models, as ANN and RF, tend to rank the genes with the capability to describe the phenomena.

From Figures 6, 8, 10, 12, and 14, Table 5 shows the fivefeature subset determined by Galgo for each of the classifiers analyzed. In each case, the subset resulting from the feature selection process causes the accuracies shown in Table 6, where can be seen that all the classifiers achieve an average accuracy higher than 0.81 , with RF being the one with the highest accuracy of 0.92 .

From Table 6, it can be observed that some of the features selected using the RF classifier also appear in other models. These features are energy, spectral spread, spectral rolloff, and MFCC2, while the MFCC 3 feature only appears in the model obtained with RF.

Table 7 shows the accuracies obtained by each classification method through the genetic algorithm, in comparison with the accuracies obtained in the previous work of feature selection by the Akaike criterion [37] and also comparing the results against classical methods of feature selection as forward selection and backward elimination. Table 7 also compares the results with the accuracies obtained by the models generated with the complete set of features. It can be observed that the feature selection process through the genetic algorithm, Galgo, performed in this work decreases by $80 \%$ the size of the original dataset, preserving the accuracy in the classification of the audios above 0.85 for four of the five classification techniques analyzed.

\section{Discussion and Conclusions}

The aim of the present work is to determine a reduced set of features, through the implementation of a selection 
TABLE 3: Recordings and audio clips per activity.

\begin{tabular}{lcccccc}
\hline \multirow{2}{*}{ Activity } & \multicolumn{2}{c}{ Generated } & \multicolumn{2}{c}{ Taken from Internet } & \multicolumn{2}{c}{ Total } \\
& Recordings & Clips & Recordings & Clips & 532 & Recordings \\
\hline Crying & 8 & 72 & 33 & 636 & 26 \\
Playing & 9 & 67 & 17 & 611 & 703 \\
Running & 9 & 81 & 30 & 652 & 39 & 692 \\
Walking & 10 & 65 & 30 & 40 & 717 \\
\hline
\end{tabular}

TABLE 4: General experimental parameter settings.

\begin{tabular}{lcc}
\hline Parameter & Value & Description \\
\hline $\begin{array}{l}\text { Data } \\
\begin{array}{l}\text { Classification } \\
\text { method }\end{array}\end{array}$ & "knn," "nearcent," "rpart," "nnet," & $\begin{array}{c}\text { Data frame containing the samples used (0 samples in columns and } \\
\text { genes in rows) }\end{array}$ \\
\hline ChromosomeSize & 5 & Methods used for the classification \\
\hline Niches & 2 & $\begin{array}{c}\text { Size of the chromosomes (the number of variables/genes included in the } \\
\text { models) }\end{array}$ \\
\hline GoalFitness & 0.90 & Number of niches (niche represents a set of chromosomes) \\
\hline MaxSolutions & 300 & Desired fitness value (fraction of correct classification) \\
\hline
\end{tabular}

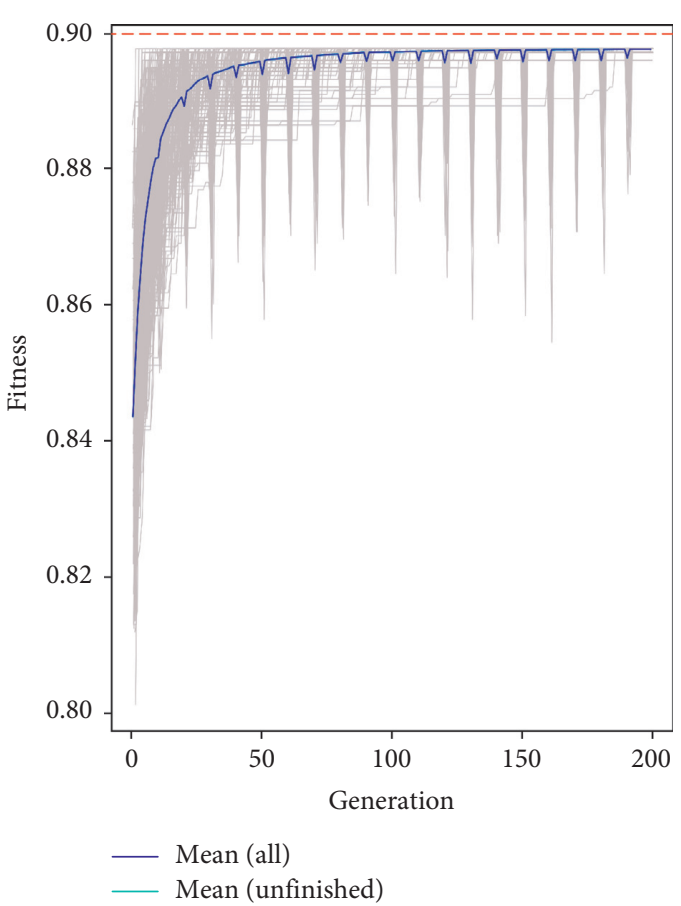

FIGURE 1: Evolution of the Galgo-generated models for the k-NN classification method.

process by a genetic algorithm, using different classification techniques, which allows the generation of a classification model of a set of children activities based on the environmental sound. Table 7 shows a comparison between the results obtained in the present work and those reported in previous works in addition to those obtained by classical feature selection techniques. In these results, it can be observed that when no feature selection technique

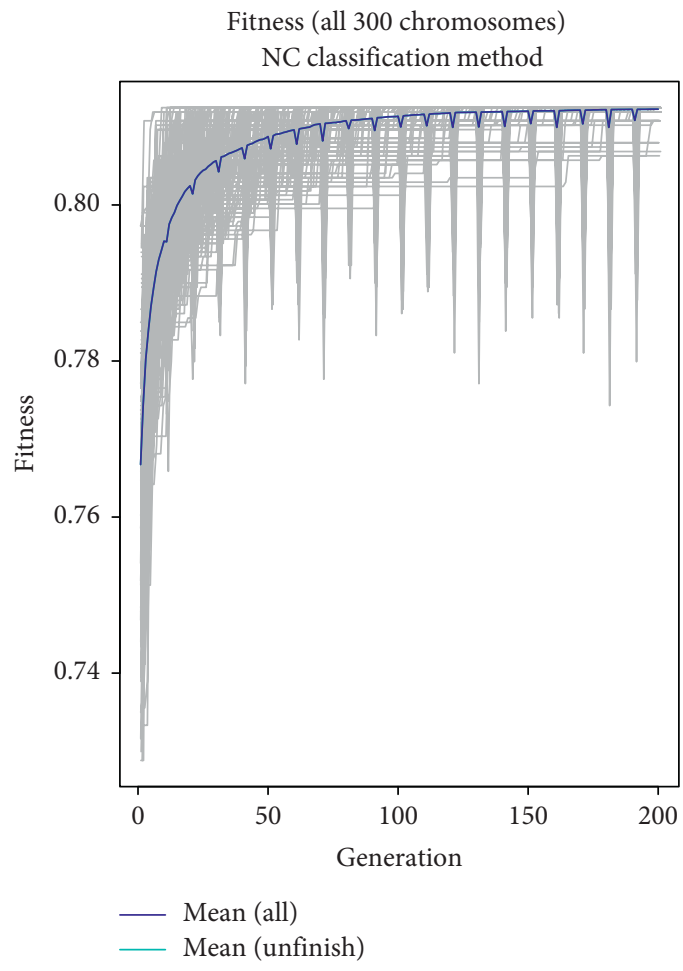

FIgURE 2: Evolution of the Galgo-generated models for the NC classification method.

is used (generating the models with the complete dataset), the k-NN classifier achieves 100\% accuracy, but the RF classifier achieves an accuracy of only $76 \%$, from which it can be concluded that the fact of using all the features does not ensure a perfect classification; in addition, k-NN seems to be prone to an over fit given the 34 features since there will be some features that do not provide relevant or 


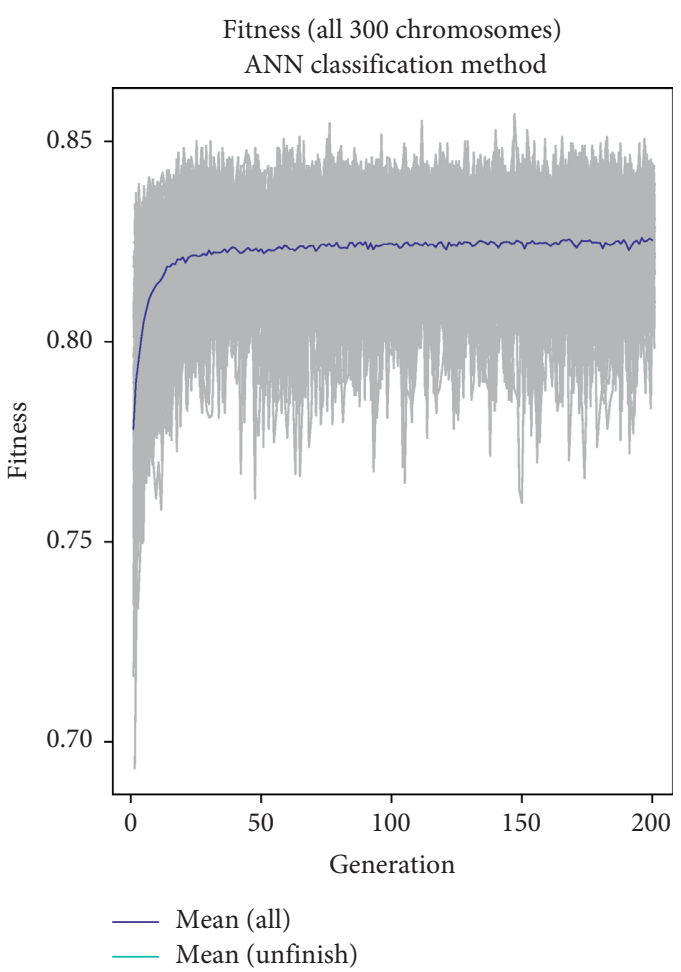

FIgURE 3: Evolution of the Galgo-generated models for the ANN classification method.

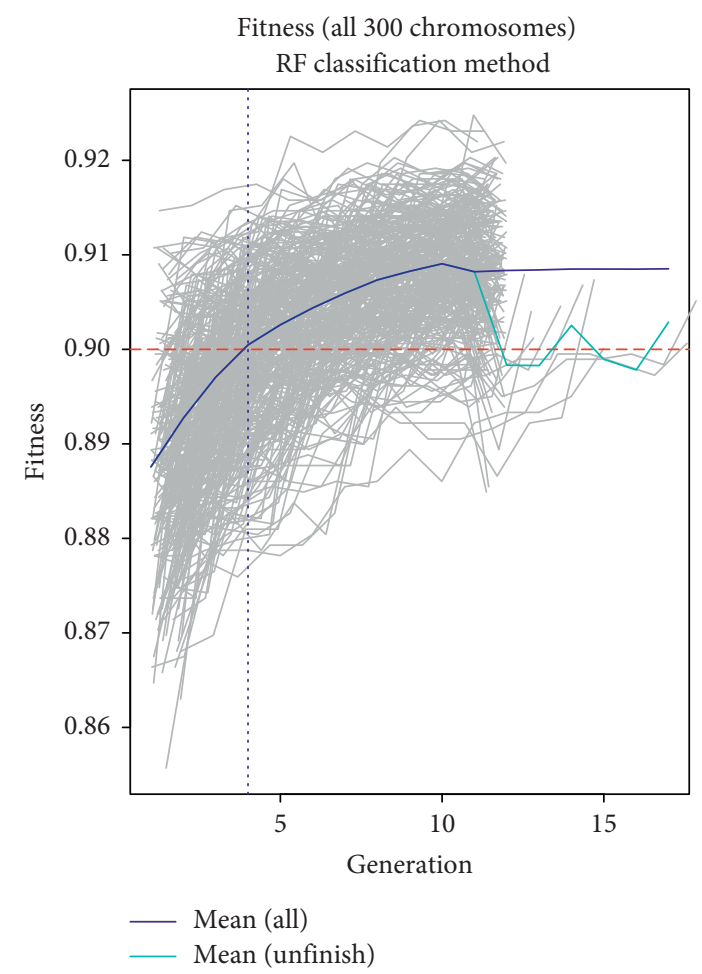

FIGURE 4: Evolution of the Galgo-generated models for the RF classification method.

redundant information for the classification, in addition to the fact that the use of more data directly impacts the processing times.

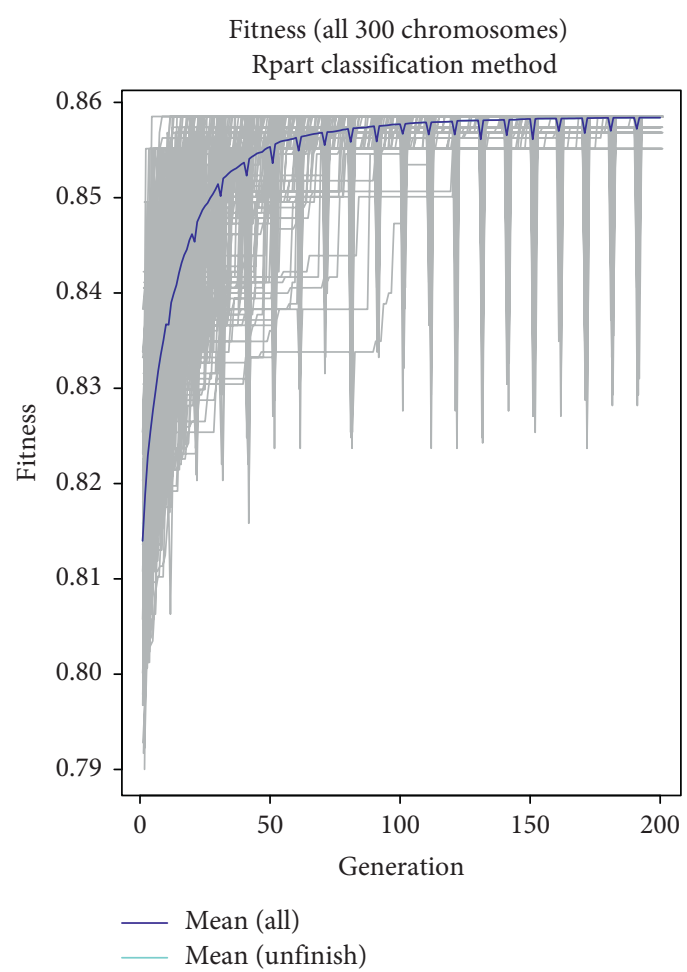

Figure 5: Evolution of the Galgo-generated models for the Rpart classification method.

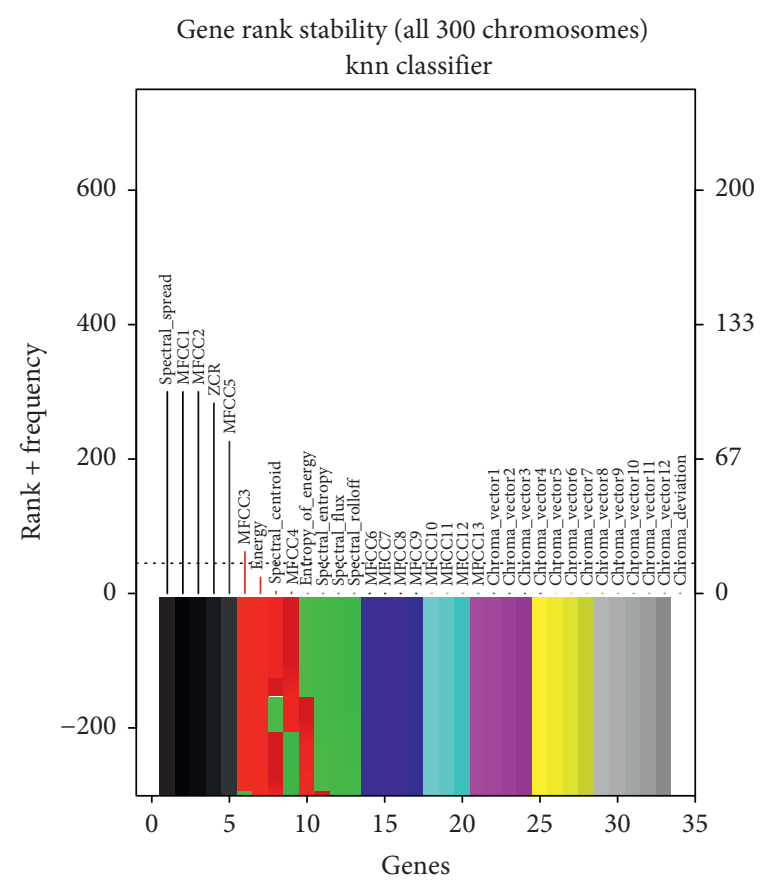

$(\%)$

FIGURE 6: Evolution of the rank with the k-NN classifier for the most frequent features.

When analyzing the results of the models that implement feature selection, when generating models of 27 features, which result from the implementation of the 


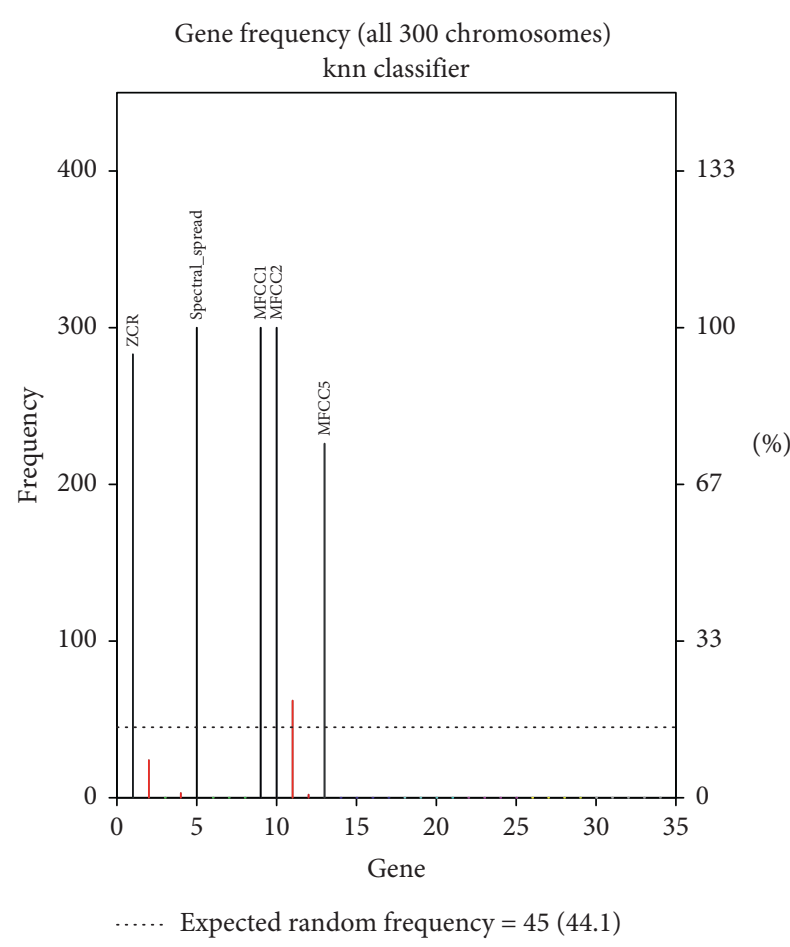

FIgURE 7: The five features chosen for the k-NN classifier according to their frequency.

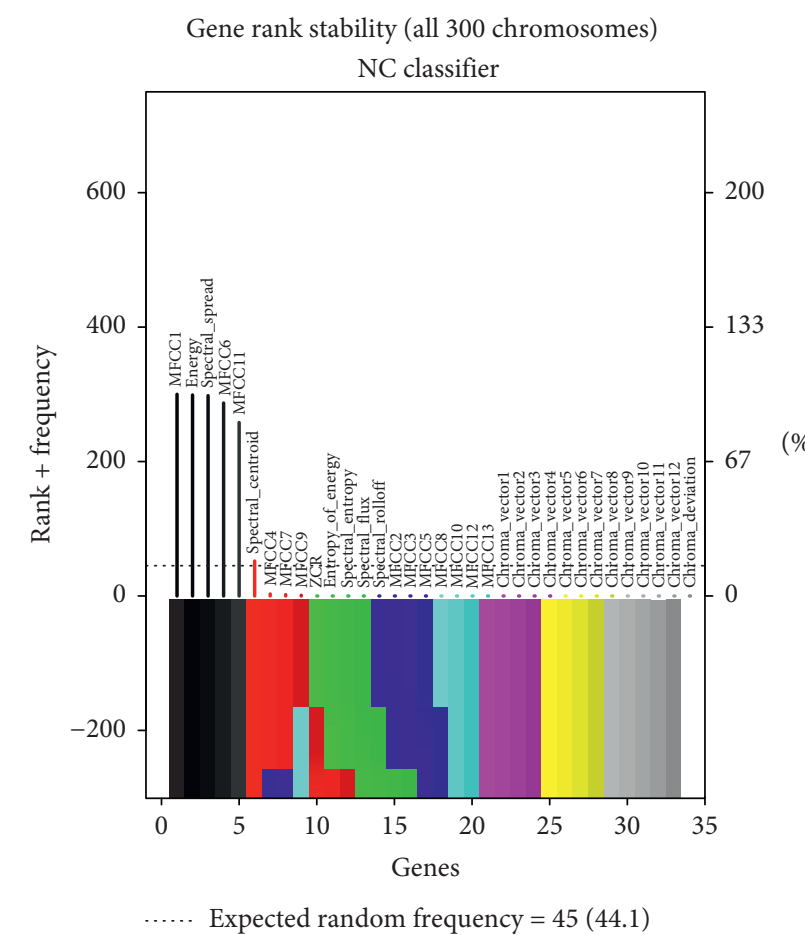

FIGURE 8: Evolution of the rank with the NC classifier for the most frequent features.

Akaike criterion selection, the classification accuracy for the RF classifier rises to $95 \%$, which means that this classifier benefited by applying feature selection, eliminating redundant data and other type of information that

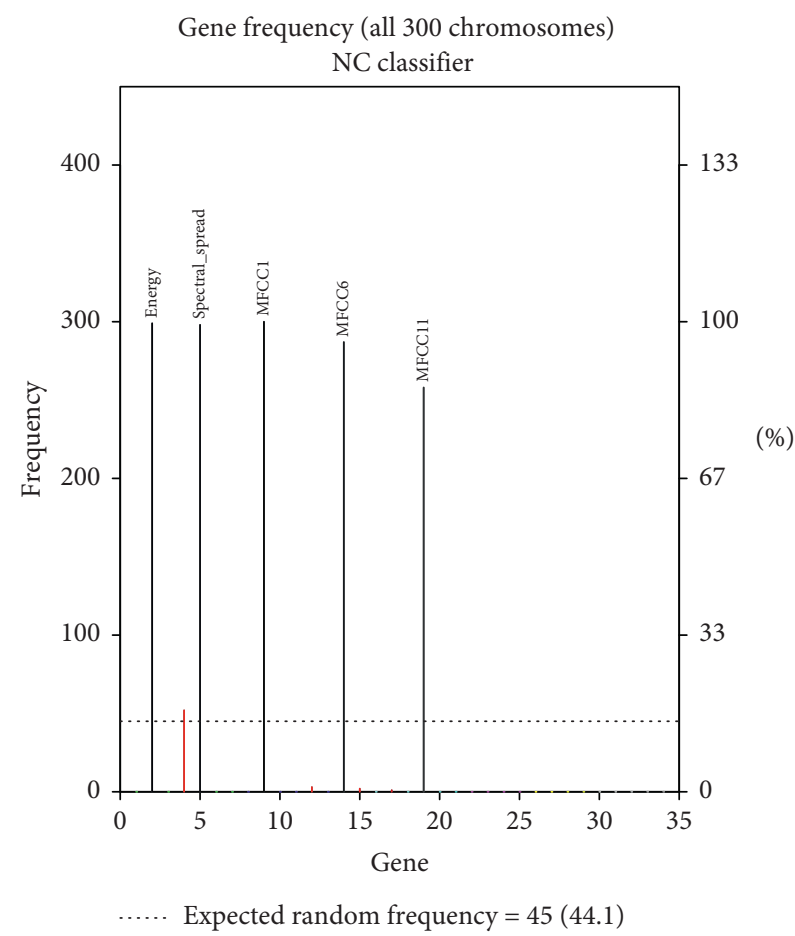

FIgURE 9: The five features chosen for the NC classifier according to their frequency.

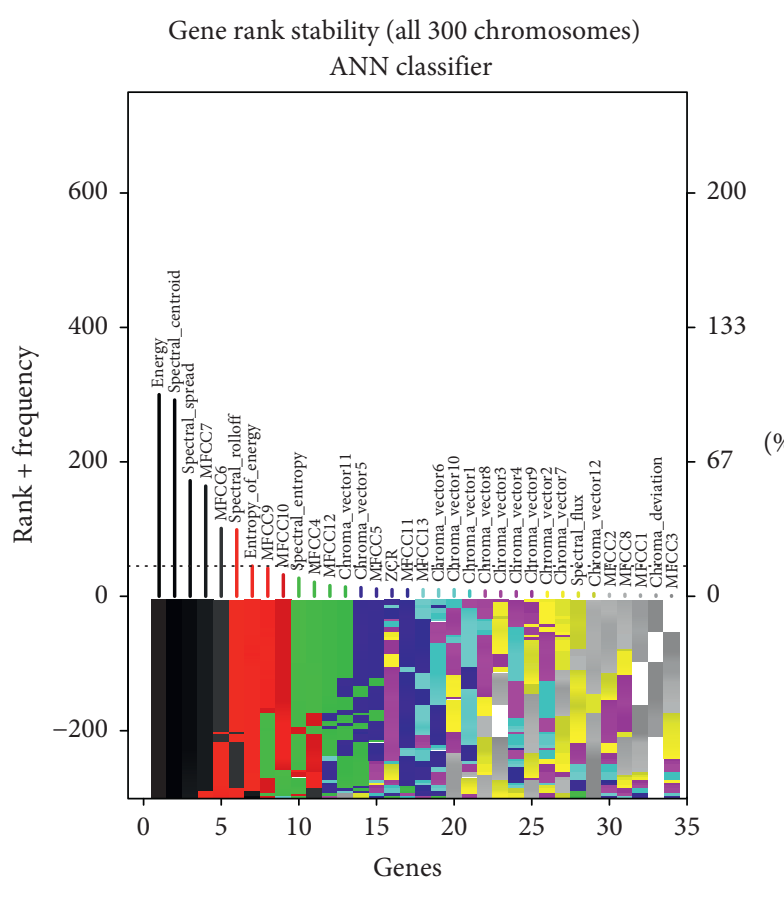

(\%)

FIGURE 10: Evolution of the rank with the ANN classifier for the most frequent features.

affected the classification, while for the k-NN classifier, the accuracy is maintained above $94 \%$, reducing the amount of data used by $20 \%$. 


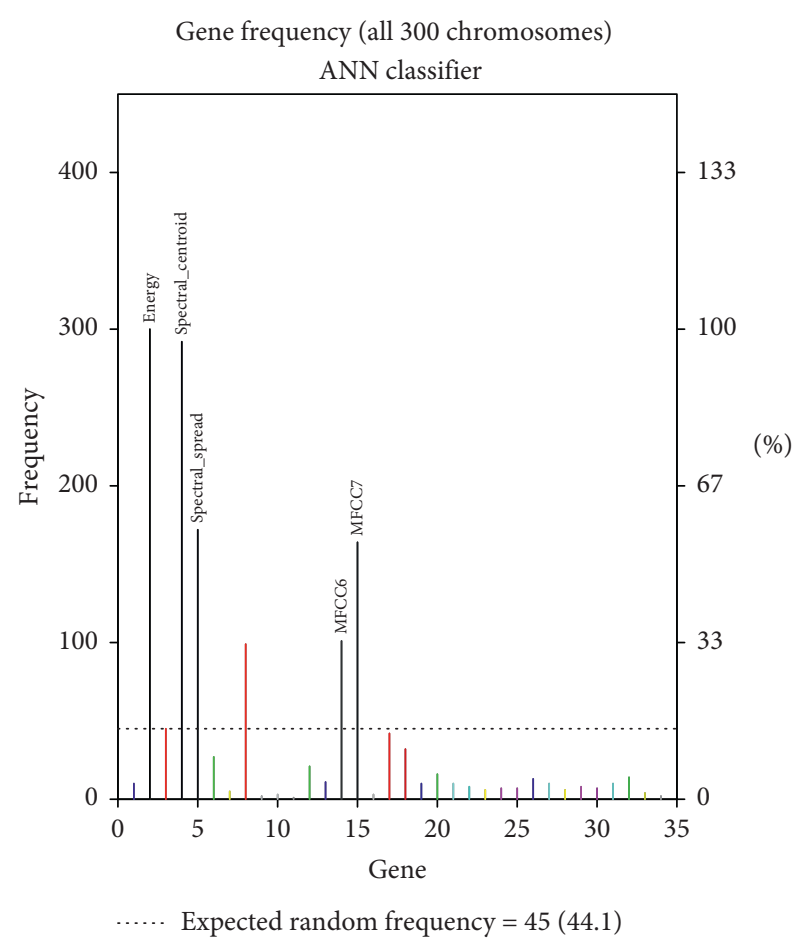

FIgURE 11: The five features chosen for the ANN classifier according to their frequency.

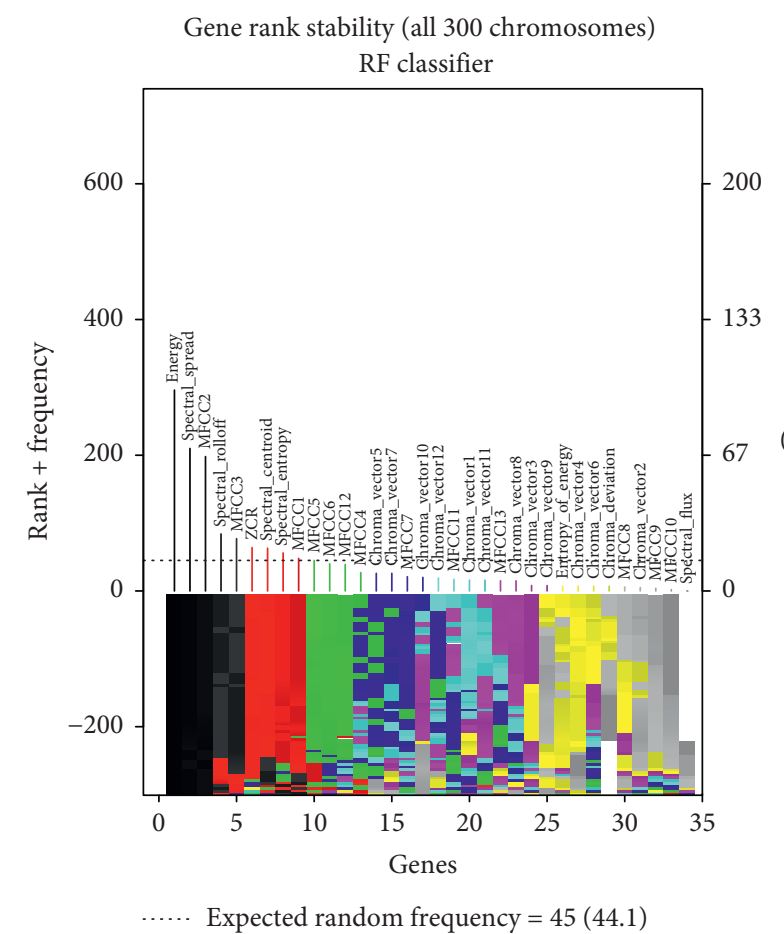

Figure 12: Evolution of the rank with the RF classifier for the most frequent features.

The classical methods of feature selection shown in the results, forward selection and backward elimination, present similar results when models with 30 features are generated,

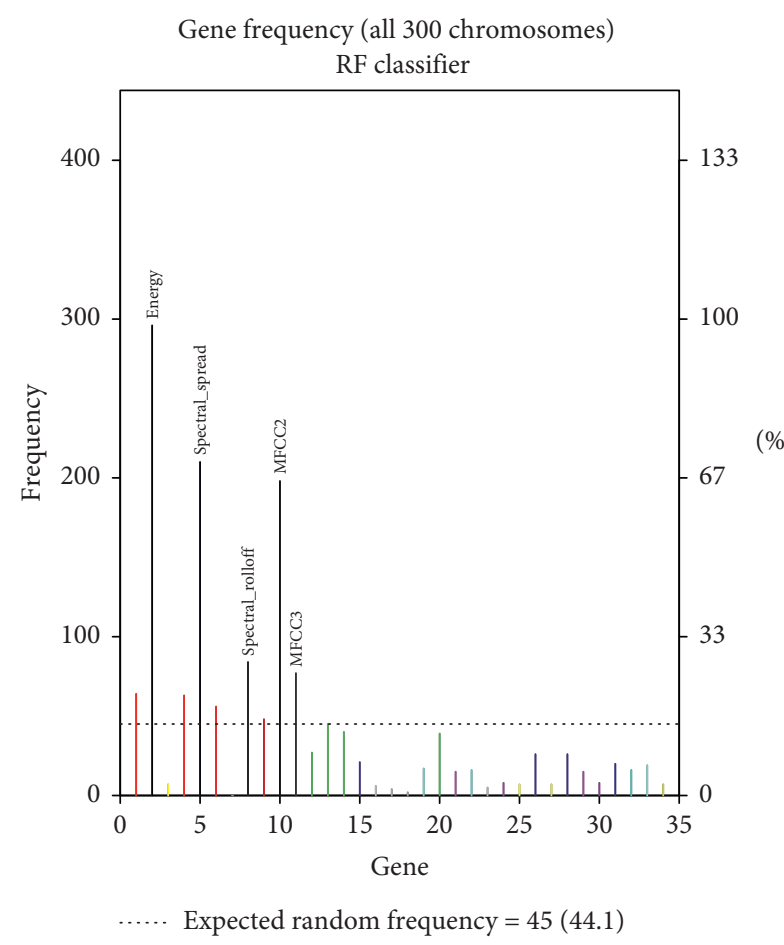

FIgURE 13: The five features chosen for the RF classifier according to their frequency.

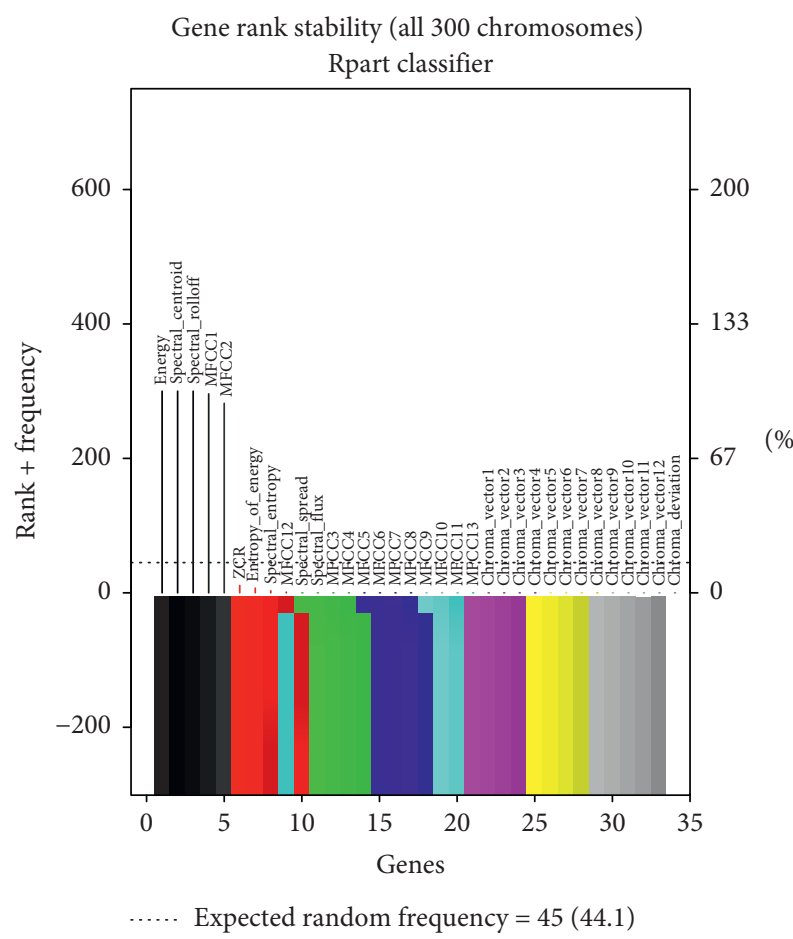

FIgURE 14: Evolution of the rank with the Rpart classifier for the most frequent features.

which reach accuracies greater than $88 \%$. While reducing the number of features to 5 , using the same methods, the accuracies are above $78 \%$, which represents a considerable 


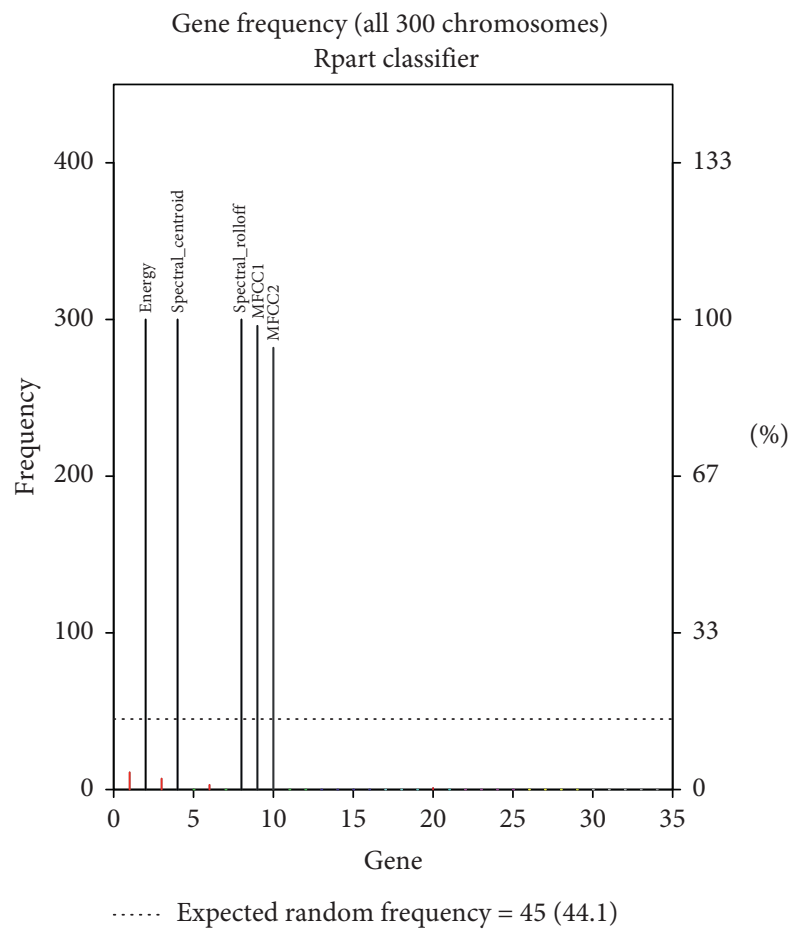

Figure 15: The five features chosen for the Rpart classifier according to their frequency.

TABle 5: Five-feature subset chosen by the genetic algorithm for each classifier.

\begin{tabular}{lc}
\hline Classifier & Features \\
\hline k-NN & ZCR, spectral spread, MFCC1, MFCC2, MFCC5 \\
NC & Energy, spectral spread, MFCC1, MFCC6, MFCC11 \\
ANN & Energy, spectral centroid, spectral spread, MFCC6, \\
MFCC7 \\
RF & Energy, spectral spread, spectral rolloff, MFCC2, \\
& MFCC3 \\
Rpart & Energy, spectral centroid, spectral rolloff, MFCC1, \\
MFCC2
\end{tabular}

TABLE 6: Average accuracy achieved by the classification methods.

\begin{tabular}{lc}
\hline Algorithm & Accuracy \\
\hline k-NN & 0.890 \\
NC & 0.815 \\
ANN & 0.850 \\
RF & 0.920 \\
Rpart & 0.857 \\
\hline
\end{tabular}

reduction in the accuracy of the classifiers, although with the benefit of the reduction in the amount of data used.

Therefore, the GA approach demonstrates an important feature reduction, only $14 \%$ of original features (5), reaching higher accuracy than the models created with more features (30 or 34) and almost the same of models with 27 features (Akaike criterion), which in terms of mobile devices means less processing power consumption needed with almost the same accuracy than much more complex models.

From GA behavior described in figure 1 to figure 15, there are several interesting points that must be mentioned:

(i) Figures 1,2 and 5, which correspond to the evolutionary process of $\mathrm{k}-\mathrm{NN}, \mathrm{NC}$, and Rpart, present a particular behavior. These low-computational cost algorithms in the mutation process include features that decrease the fitness of the model, and finally these features are excluded almost immediately from the selection.

(ii) In Figures 7, 9 and 15, the comportment described above is remarked, almost equivalent frequency of the finally selected features for each of the algorithms.

(iii) Otherwise, it is shown in Figures 3 and 4 that algorithms such as RF and ANN have a smooth evolutionary process, given the capabilities to find more complex relationship between features.

Hence, from the results obtained in Section 3, it can be concluded that the five-feature sets determined by the genetic algorithm used achieve similar accuracies to the models generated using the original set of features, but with the advantage that there is an $80 \%$ reduction in the dataset size, including all its implications, such as a faster data processing, which is very important when working with applications for mobile devices since its resources are limited. 
TABLE 7: Accuracies by the generated model.

\begin{tabular}{|c|c|c|c|}
\hline Number of features & Feature selection method & Classifier & Accuracy \\
\hline 5 & Genetic algorithm & $\mathrm{k}-\mathrm{NN}$ & 0.89 \\
\hline 5 & Genetic algorithm & NC & 0.815 \\
\hline 5 & Genetic algorithm & ANN & 0.85 \\
\hline 5 & Genetic algorithm & $\mathrm{RF}$ & 0.92 \\
\hline 5 & Genetic algorithm & Rpart & 0.857 \\
\hline 27 & Akaike criterion & $\mathrm{k}-\mathrm{NN}$ & 0.9425 \\
\hline 27 & Akaike criterion & $\mathrm{RF}$ & 0.9525 \\
\hline 34 & None & $\mathrm{k}-\mathrm{NN}$ & 1.00 \\
\hline 34 & None & RF & 0.7637 \\
\hline 5 & Forward selection & SVM & 0.7944 \\
\hline 5 & Forward selection & $\mathrm{k}-\mathrm{NN}$ & 0.8611 \\
\hline 5 & Forward selection & $\mathrm{RF}$ & 0.8917 \\
\hline 5 & Forward selection & Gradient boosting & 0.8710 \\
\hline 5 & Backward elimination & SVM & 0.8079 \\
\hline 5 & Backward elimination & $\mathrm{k}-\mathrm{NN}$ & 0.8545 \\
\hline 5 & Backward elimination & $\mathrm{RF}$ & 0.8946 \\
\hline 5 & Backward elimination & Gradient boosting & 0.8797 \\
\hline \multirow[t]{2}{*}{30} & Forward selection & SVM & 0.8867 \\
\hline & Backward elimination & & \\
\hline \multirow[t]{2}{*}{30} & Forward selection & $\mathrm{k}-\mathrm{NN}$ & 0.9075 \\
\hline & Backward elimination & & \\
\hline \multirow[t]{2}{*}{30} & Forward selection & $\mathrm{RF}$ & 0.9208 \\
\hline & Backward elimination & & \\
\hline \multirow[t]{2}{*}{30} & Forward selection & Gradient boosting & 0.9228 \\
\hline & Backward elimination & & \\
\hline
\end{tabular}

\section{Future Work}

The present work allows us to demonstrate that it is possible to generate children activity classification models using environmental sound by applying feature selection through genetic algorithms, preserving accuracies greater than $81 \%$ and reducing the set of data used by $85 \%$; however, some aspects can be worked on in the future. The proposed future work is as follows:

(i) To work with a different set of features. To extract a larger feature set from the audio files to verify the behavior of the feature selection methods now analyzed with a different set of features and contrast the results with those obtained in this work to finally determine the subset of features that best describes the activities.

(ii) To combine feature selection methods. Generate classification models using subsets of features resulting from the combination of the method proposed in the present work with the classical methods of feature selection such as forward selection and backward elimination.

\section{Data Availability}

Open data files are available at https://ingsoftware.reduaz.mx/amidami

\section{Conflicts of Interest}

The authors declare that they have no conflicts of interest.

\section{References}

[1] M. G. Navarro, "Inteligencia ambiental: entornos inteligentes ante el desafío de los procesos inferenciales," Eidos: Revista de Filosofía, vol. 15, pp. 184-205, 2011.

[2] D. Ding, R. A. Cooper, P. F. Pasquina, and L. Fici-Pasquina, "Sensor technology for smart homes," Maturitas, vol. 69, no. 2, pp. 131-136, 2011.

[3] A. H. Buckman, M. Mayfield, and S. B. M. Beck, "What is a smart building?" Smart and Sustainable Built Environment, vol. 3, no. 2, pp. 92-109, 2014.

[4] H. Chourabi, T. Nam, S. Walker et al., "Understanding smart cities: an integrative framework," in Proceedings of the 2012 45th Hawaii International Conference on System Sciences, Maui, HI, USA, January 2012.

[5] Y. Kim and H. Ling, "Human activity classification based on micro-Doppler signatures using a support vector machine," IEEE Transactions on Geoscience and Remote Sensing, vol. 47, no. 5, pp. 1328-1337, 2009.

[6] A. Mannini and A. M. Sabatini, "Accelerometry-based classification of human activities using markov modeling," Computational Intelligence and Neuroscience, vol. 2011, Article ID 647858, 10 pages, 2011.

[7] M. Zenaldin and R. M. Narayanan, "Radar micro-Doppler based human activity classification for indoor and outdoor environments," in Proceedings of the Radar Sensor Technology XX, Baltimore, MD, USA, May 2016.

[8] M. Maciejewska, A. Szczurek, and A. Dolega, "Classification of human activities indoors using microclimate sensors and semiconductor gas sensors," in Proceedings of the 8th International Conference on Sensor Networks, Prague, Czech Republic, February 2019.

[9] S. Walke, S. Marne, O. Langhe, A. Kulkarni, and S. Mahajan, "Human detection and monitoring system using multi sensor data fusion," in Proceedings of the 2018 Second International 
Conference on Intelligent Computing and Control Systems (ICICCS), Madurai, India, June 2018.

[10] A. Bayat, M. Pomplun, and D. A. Tran, "A study on human activity recognition using accelerometer data from smartphones," Procedia Computer Science, vol. 34, pp. 450-457, 2014.

[11] H. Medjahed, D. Istrate, J. Boudy, and B. Dorizzi, "Human activities of daily living recognition using fuzzy logic for elderly home monitoring," in Proceedings of the 2009 IEEE International Conference on Fuzzy Systems, Jeju Island, South Korea, August 2009.

[12] S. Chernbumroong, S. Cang, A. Atkins, and H. Yu, "Elderly activities recognition and classification for applications in assisted living," Expert Systems with Applications, vol. 40, no. 5, pp. 1662-1674, 2013.

[13] S. Boughorbel, J. Breebaart, F. Bruekers, I. Flinsenberg, and W. T. Kate, "Child-activity recognition from multi-sensor data," in Proceedings of the 7th International Conference on Methods and Techniques in Behavioral Research-MB 10, Eindhoven, Netherlands, August 2010.

[14] Y. Nam and J. W. Park, "Child activity recognition based on cooperative fusion model of a triaxial accelerometer and a barometric pressure sensor," IEEE Journal of Biomedical and Health Informatics, vol. 17, no. 2, pp. 420-426, 2013.

[15] L. Piyathilaka and S. Kodagoda, "Human activity recognition for domestic robots," Springer Tracts in Advanced Robotics Field and Service Robotics, pp. 395-408, Springer, Cham, Switzerland, 2015.

[16] P. Arnon, "Classification model for multi-sensor data fusion apply for human activity recognition," in Proceedings of the 2014 International Conference on Computer, Communications, and Control Technology (I4CT), Langkawi, Malaysia, September 2014.

[17] O. D. Lara and M. A. Labrador, "A survey on human activity recognition using wearable sensors," IEEE Communications Surveys \& Tutorials, vol. 15, no. 3, pp. 1192-1209, 2013.

[18] S. Kurashima and S. Suzuki, "Improvement of activity recognition for child growth monitoring system at kindergarten," in Proceedings of the IECON 2015-41st Annual Conference of the IEEE Industrial Electronics Society, Yokohama, Japan, November 2015.

[19] T. L. Westeyn, G. D. Abowd, T. E. Starner, J. M. Johnson, P. W. Presti, and K. A. Weaver, "Monitoring children's developmental progress using augmented toys and activity recognition," Personal and Ubiquitous Computing, vol. 16, no. 2, pp. 169-191, 2011.

[20] J. Wu, S. Guo, H. Huang, W. Liu, and Y. Xiang, "Information and communications technologies for sustainable development goals: state-of-the-art, needs and perspectives," IEEE Communications Surveys \& Tutorials, vol. 20, no. 3, pp. 2389-2406, 2018.

[21] C. E. Galván-Tejada, J. I. Galván-Tejada, J. M. Celaya-Padilla et al., "An analysis of audio features to develop a human activity recognition model using genetic algorithms, random forests, and neural networks," Mobile Information Systems, vol. 2016, Article ID 1784101, 10 pages, 2016.

[22] J. M. Sim, Y. Lee, and O. Kwon, "Acoustic sensor based recognition of human activity in everyday life for smart home services," International Journal of Distributed Sensor Networks, vol. 11, no. 9, Article ID 679123, 2015.

[23] R. Atat, L. Liu, J. Wu, G. Li, C. Ye, and Y. Yang, "Big data meet cyber-physical systems: a panoramic survey," IEEE Access, vol. 6, pp. 603-636, 2018.
[24] V. Trevino and F. Falciani, "Galgo: an r package for multivariate variable selection using genetic algorithms," Bioinformatics, vol. 22, no. 9, pp. 1154-1156, 2006.

[25] M. Kose, O. D. Incel, and C. Ersoy, "Online human activity recognition on smart phones," in Proceedings of the Workshop on Mobile Sensing: From Smartphones and Wearables to Big Data, vol. 16, pp. 11-15, Beijing, China, April 2012.

[26] O. Masoud and N. Papanikolopoulos, "Recognizing human activities," in Proceedings of the IEEE Conference on Advanced Video and Signal Based Surveillance, 2003, pp. 157-162, IEEE, Miami, FL, USA, July 2003.

[27] J. Parkka, M. Ermes, P. Korpipaa, J. Mantyjarvi, J. Peltola, and I. Korhonen, "Activity classification using realistic data from wearable sensors," IEEE Transactions on Information Technology in Biomedicine, vol. 10, no. 1, pp. 119-128, 2006.

[28] P. Casale, O. Pujol, and P. Radeva, "Human activity recognition from accelerometer data using a wearable device," in Pattern Recognition and Image Analysis, pp. 289-296, Springer, Berlin, Germany, 2011.

[29] C. Strobl, J. Malley, and G. Tutz, "An introduction to recursive partitioning: rationale, application, and characteristics of classification and regression trees, bagging, and random forests," Psychological Methods, vol. 14, no. 4, pp. 323-348, 2009.

[30] C. Galván-Tejada, J. García-Vázquez, and R. Brena, "Magnetic field feature extraction and selection for indoor location estimation," Sensors, vol. 14, no. 6, pp. 11 001-011 015, 2014.

[31] C. Rojas-Guzman and M. A. Kramer, "Remote diagnosis and monitoring of complex industrial systems using a genetic algorithm approach," in Proceedings of 1994 IEEE International Symposium on Industrial Electronics (ISIE'94), pp. 363-367, IEEE, Santiago, Chile, May 1994.

[32] C. D. Luna-Gómez, L. A. Zanella-Calzada, M. A. AcostaGarcía, J. I. Galván-Tejada, C. E. Galván-Tejada, and J. M. Celaya-Padilla, "Can multivariate models based on moaks predict oa knee pain? data from the osteoarthritis initiative," in Proceedings of the Medical Imaging 2017: Computer-Aided Diagnosis, vol. 10134, International Society for Optics and Photonics, Orlando, FL, USA, February 2017.

[33] C. Galván-Tejada, L. Zanella-Calzada, J. Galván-Tejada et al., "Multivariate feature selection of image descriptors data for breast cancer with computer-assisted diagnosis," Diagnostics, vol. 7, no. 1, p. 9, 2017.

[34] D. M. Blanco-Murillo, A. García-Domínguez, C. E. GalvánTejada, and J. Celaya-Padilla, "Comparación del nivel de precisión de los clasificadores support vector machines, $\mathrm{k}$ nearest neighbors, random forests, extra trees y gradient boosting en el reconocimiento de actividades infantiles utilizando sonido ambiental," Research in Computing Science, vol. 147, no. 5, 2018.

[35] G. Chandrashekar and F. Sahin, "A survey on feature selection methods," Computers \& Electrical Engineering, vol. 40, no. 1, pp. 16-28, 2014.

[36] Welcome to python.org, https://www.python.org/.

[37] A. García-Domínguez and C. E. Galván-Tejada, "Reconocimiento de actividades infantiles utilizando sonido ambiental: un enfoque preliminar," Research in Computing Science, vol. 139, pp. 71-79, 2017.

[38] E. D. Scheirer, "Tempo and beat analysis of acoustic musical signals," The Journal of the Acoustical Society of America, vol. 103, no. 1, pp. 588-601, 1998.

[39] H. Wang, A. Divakaran, A. Vetro, S.-F. Chang, and H. Sun, "Survey of compressed-domain features used in audio-visual indexing and analysis," Journal of Visual Communication and Image Representation, vol. 14, no. 2, pp. 150-183, 2003. 
[40] G. Tzanetakis and P. Cook, "IEEE transactions on speech and audio processing publication information," IEEE Transactions On Speech And Audio Processing, vol. 13, no. 3, p. c2, 2005.

[41] E. Aarts, J. Korst, and W. Verhaegh, "Algorithms in ambient intelligence," Ambient Intelligence, pp. 349-373, Springer, Berlin, Germany, 2005.

[42] J. A. Stork, L. Spinello, J. Silva, and K. O. Arras, "Audio-based human activity recognition using non-markovian ensemble voting," in Proceedings of the 2012 IEEE RO-MAN: The 21st IEEE International Symposium on Robot and Human Interactive Communication, Paris, France, September 2012.

[43] M. Markakis, Selection of relevant features for audio classification tasks, Ph.D thesis, University of Crete, Heraklion, Greece, 2011.

[44] M. Mascia, A. Canclini, F. Antonacci, M. Tagliasacchi, A. Sarti, and S. Tubaro, "Forensic and anti-forensic analysis of indoor/outdoor classifiers based on acoustic clues," in Proceedings of the 2015 23rd European Signal Processing Conference (EUSIPCO), Aalborg, Denmark, August 2015.

[45] R. R Core Team, A Language and Environment for Statistical Computing, R Foundation for Statistical Computing, Vienna, Austria, 2019, https://www.R-project.org/.

[46] P. Thanh Noi and M. Kappas, "Comparison of random forest, $\mathrm{k}$-nearest neighbor, and support vector machine classifiers for land cover classification using sentinel-2 imagery," Sensors, vol. 18 , no. 1 , p. $18,2018$.

[47] A. R. Chowdhury, T. Chatterjee, and S. Banerjee, "A random forest classifier-based approach in the detection of abnormalities in the retina," Medical \& Biological Engineering \& Computing, vol. 57, no. 1, pp. 193-203, 2019.

[48] L. Breiman, "Random forests," Machine Learning, vol. 45, no. 1, pp. 5-32, 2001.

[49] X. Zhu, X. Du, M. Kerich, F. W. Lohoff, and R. Momenan, "Random forest based classification of alcohol dependence patients and healthy controls using resting state mri," Neuroscience Letters, vol. 676, pp. 27-33, 2018.

[50] F. Vekeman, J. E. Piña-Garza, W. Y. Cheng et al., "Development of a classifier to identify patients with probable lennox-gastaut syndrome in health insurance claims databases via random forest methodology," Current Medical Research and Opinion, vol. 35, no. 8, pp. 1415-1420, 2019.

[51] L. Zanella-Calzada, C. Galván-Tejada, N. Chávez-Lamas et al., "Deep artificial neural networks for the diagnostic of caries using socioeconomic and nutritional features as determinants: data from NHANES 2013-2014," Bioengineering, vol. 5, no. 2, p. 47, 2018.

[52] J.-X. Lin, Z.-K. Wang, W. Wang et al., "Development and validation of a new staging system for node-negative gastric cancer based on recursive partitioning analysis: an international multi-institutional study," Cancer Medicine, vol. 8, no. 6, pp. 2962-2970, 2019.

[53] V. Trevino and F. Falciani, "Galgo: genetic algorithms for multivariate statistical models from large-scale functional genomics data," 2018, https://CRAN.R-project.org/ package $=$ galgo. 